\title{
La medicalización de los problemas sociales
}

\section{Medicalization of social problems}

\section{Dr. C. Teresa Forcades i Vila}

Monasterio de Sant Benet de Montserrat. Cataluña, España.

\section{RESUMEN}

El presente artículo analiza el tema de la medicalización de los problemas sociales como una extensión indebida del modelo médico. Esta medicalización tiene una triple consecuencia negativa que se caracteriza como: némesis metodológica, némesis clínica y némesis social. Se aportan ejemplos de la literatura médica contemporánea y se profundiza particularmente en la medicalización de la violencia social y en el abuso ideológico de la ciencia que esta demuestra tanto en el pasado como, lamentablemente, también en la actualidad.

Palabras clave: medicalización, despolitización, yatrogenia, némesis médica, violencia social, Illich, Cartwright, post hoc propter hoc, límites metodológicos, disestesia etiópica, drapetomanía.

\section{ABSTRACT}

The present article analyzed the topic of medicalization of social problems as an inappropriate extension of the medical model that brings about three negative consequences: methodological nemesis, clinical nemesis and social nemesis. Several examples were drawn from the current medical literature, with a special emphasis on the medicalization of social violence and the ideological misuse of science in the past and, unfortunately, at present.

Key words: medicalization, desocialization, iatrogeny, medical nemesis, social violence, Illich, Cartwright, post hoc propter hoc, methodological limits, ethiopic dysethesia, drapetomania. 
En el año 2004, el International J ournal of Epidemiology, una de las principales revistas en epidemiología, publicó un estudio realizado en Noruega que demuestra que, al menos en cuanto a la población de aquel país se refiere, existe una asociación entre el peso al nacer y la posibilidad que quedarse sin trabajo en la vida adulta. ${ }^{1}$ Unos años antes, en 2001 , otro equipo de investigadores había descubierto una correlación entre el bajo peso al nacer y el hecho de estar soltero en la vida adulta. ${ }^{2}$ Las respuestas críticas, desde dentro de la misma profesión médica y entre los mismos investigadores, no se hicieron esperar. ${ }^{3}$ Los argumentos en contra de este tipo de estudios se pueden resumir en tres puntos: némesis metodológica, yatrogenia o némesis clínica y némesis social.

\section{NÉMESIS METODOLÓGICA}

I van Illich aplicó en 1974 la noción de némesis (venganza o castigo por haber confiado en exceso en los propios recursos) al ámbito médico. ${ }^{4}$ Retomando su nomenclatura, podemos denominar "némesis metodológica" a la posibilidad que confiando de forma excesiva en una herramienta tan útil como es la estadística, obtengamos resultados que pueden tener consecuencias negativas graves, como por ejemplo la estigmatización de las personas que nacen con bajo peso. La némesis en el sentido que le dio Illich no tiene nada que ver con la negligencia o el error. Es obvio que un profesional de la salud puede equivocarse y puede cometer una negligencia que al paciente le cueste muy cara, y es igualmente obvio que un investigador puede equivocarse en la aplicación de las herramientas propias de su trabajo, incluida la estadística. La noción de némesis no hace referencia a esta posibilidad casual que es imposible de eliminar (el error humano), sino a una limitación de tipo intrínseco, a una posibilidad de hacer daño inherente al sistema médico moderno: la némesis sería un mal que va en aumento a medida que avanza la medicalización, un mal que crece inevitablemente a medida que insistimos en aproximarnos a la realidad de la enfermedad y la salud desde un modelo teórico que no tiene suficientemente en cuenta los propios límites. Para ilustrar los límites propios de la estadística se suele proponer el caso hipotético de un pueblo en el cual la mitad de la población comiera dos pollos al día y la otra mitad no comiera ninguno; si analizamos la media estadística, podemos llegar a la conclusión de que en este pueblo todo el mundo come un pollo al día. Este es un ejemplo banal, puesto que la ciencia estadística tiene una manera muy simple (el cálculo de la varianza muestral), de evitar este error básico, pero cuando nos situamos a un nivel más avanzado de análisis las cosas no son ni mucho menos tan sencillas. La misma revista de epidemiología donde apareció el artículo sobre el estudio noruego, publicó a continuación en el mismo número una respuesta crítica que consideraba que el análisis de regresión múltiple que habían usado los investigadores noruegos era inadecuado, y que si hubieran usado un modelo de equivalencia estructural los resultados habrían sido otros. ${ }^{3}$ ¿Tiene razón este crítico? ¿O tienen razón los autores del artículo original? Las matemáticas son una ciencia exacta, pero su aplicación a las ciencias sociales no lo es. Detrás de cada estudio hay una serie de decisiones que están influidas por factores personales, culturales, políticos, económicos [...] a veces simplemente cronológicos (falta de tiempo para estudiar un modelo estadístico con el cual no se está familiarizado) o de amistad (escribir un artículo conjuntamente con un colega que necesita una promoción y que domina unas determinadas herramientas estadísticas y no otras). La némesis metodológica tiene lugar únicamente cuando olvidamos esta contingencia y tomamos los resultados de un estudio estadístico como si fueran verdades absolutas. El problema no es que la metodología científica tenga límites, sino que no los reconozcamos adecuadamente y pretendamos basar las decisiones en salud pública únicamente o principalmente en resultados estadísticos. La némesis metodológica es la distorsión de la realidad que conocemos con el nombre de "pseudociencia" o "cientifismo" (equivale al "saber disciplinar" en nomenclatura del filósofo M. Foucault y 
al "discurso de la universidad" en nomenclatura del psicoanalista Jacques Lacan). Es importante distinguir la asociación de la causalidad: que dos variables estén asociadas (cómo por ejemplo el bajo peso y la tasa de paro) no implica que haya entre ellas una relación de causa efecto; no tener en cuenta esta precaución básica es incurrir en la falacia post hoc, ergo propter hoc (la falacia de creer que si una realidad acontece a continuación de otra puede deducirse que la primera es la causa de la segunda). Esta falacia se enseña en el primer curso de estadística. Se enseña y se sabe, pero su conocimiento no evita el cientifismo porque asociación no equivale a causalidad, pero tampoco la descarta. Este es el problema. Los paquetes estadísticos actuales son tan potentes que permiten el análisis de variables muy complejas en un tiempo récord y son capaces de descubrir por casualidad las asociaciones más inusitadas. El hecho de afirmar como cierta una relación de causalidad entre dos variables asociadas temporalmente sea una falacia, no implica que esta causalidad no sea posible. No la podemos afirmar como cierta, pero -y este es el factor decisivo en la prácticatampoco la podemos descartar usando la metodología científica. Es por eso que, una vez establecida la asociación estadística entre el bajo peso al nacer y la posibilidad de quedarse sin trabajo o de quedarse soltero en la vida adulta, puede darse el caso ( $y$ esto es de hecho lo que ocurre habitualmente), que la sociedad o los dirigentes políticos o los responsables de la salud pública de un determinado país, lleguen a la conclusión de que hay que hacer algo para ayudar a las personas supuestamente afectadas. Y aquí empieza la medicalización y la yatrogenia o némesis clínica que le es propia.

\section{YATROGENI A O NÉMESI S CLÍ NI CA}

El mero hecho de que un estudio publicado en una revista de prestigio establezca una asociación estadística en principio tan absurda como la supuesta asociación existente entre haber nacido con bajo peso y quedarse en el paro o ser soltero, puede activar el sistema médico actual de forma que se acaben proponiendo intervenciones concretas a fin de prevenir o tratar el supuesto problema como si fuera cierto. "Más vale prevenir que curar", se arguye. Las intervenciones propuestas pueden ser diversas: regímenes alimenticios, cursos de capacitación profesional o social, acompañamiento psicológico para los padres y los hijos afectados [...], en cualquier caso, el resultado será la producción de una nueva etiqueta diagnóstica y de un nuevo grupo de afectados que interpretarán en términos médicos el hecho de estar en el paro o de no haber encontrado pareja. Las diferentes intervenciones médicas pueden tener efectos negativos sobre las personas que se sometan a ellas, especialmente si los tratamientos propuestos son de tipo farmacológico. Los efectos negativos de las intervenciones médicas constituyen la yatrogenia o némesis clínica.

\section{NÉMESI S SOCIAL}

Existe, por último, un tercer nivel de némesis médica o de efectos negativos de la medicalización: tomarse seriamente la posibilidad de que el paro sea consecuencia del bajo peso al nacer, además de suponer un abuso de la estadística como herramienta capaz de aproximarnos a la realidad (némesis metodológica o cientifista) y además de someter a unas determinadas personas a unas intervenciones con posibles efectos secundarios graves (némesis clínica), puede desviar la atención y los recursos de las verdaderas causas del problema del paro o puede conceptualizar como problema algo que por sí mismo no tiene por qué serlo e incluso puede ser algo positivo (no estar casado). En el caso del paro se puede producir una despolititzación: la responsabilidad del problema del paro pasa a recaer sobre el individuo y se puede llegar a cuestionar que la organización socioeconómica o la toma de decisiones políticas sean un factor relevante. 
La mentalidad científica tiene que estar por definición máximamente abierta a las sorpresas y a las causalidades inesperadas que hacen avanzar nuestra manera de pensar sobre los problemas y sobre la vida misma. En este sentido, la posibilidad de que el bajo peso al nacer sea un factor causal significativo para quedarse sin trabajo o para quedarse soltero, no hace falta que sea descartada de forma absoluta. No se trata de sustituir un dogmatismo (el resultado estadístico interpretado como verdad absoluta) por otro (la absolutitzación de la manera actual de comprender los problemas y el mundo), sino de tomar conciencia de que la responsabilidad de interpretar la realidad y de tomar decisiones a nivel individual y colectivo no se puede delegar en ningún equipo de expertos, por más alta calificación académica que tengan.

También tenía la más alta calificación académica el Dr. Samuel A. Cartwright cuando creyó descubrir en 1851 que una circunstancia de su tiempo considerada hasta entonces - como es el caso del paro en nuestros días- de origen social, tenía en realidad una causa médica. La circunstancia en cuestión era la poca motivación por el trabajo y la falta de diligencia en general que tenían algunos esclavos de las plantaciones del sur de los EE. UU., especialmente de las que se encontraban cerca de la frontera con alguno de los estados abolicionistas norteños. El Dr. Cartwright postuló que la causa de esta falta de motivación era un transtorno mental que denominó "disestesia etiópica". ${ }^{5}$ Así como la palabra "an-estesia" hace referencia a la falta de sensibilidad, Cartwright postuló la palabra "dis-estesia"para describir una sensibilidad anómala, en este caso disminuida; la palabra "etiópica" se explica porque este adjetivo se usaba en la época como sinónimo de "africano". Cartwright explicó que este transtorno estaba asociado a unas lesiones características de la piel, causadas por las heridas o quemaduras que el paciente se hacía a si mismo:

Su cuerpo se encuentra invadido por la torpeza y la insensibilidad; los nervios sensitivos distribuidos por la piel pierden la sensibilidad en un grado tan acusado que a menudo el paciente llega a quemarse sin darse cuenta en el fuego al lado del cual pasa las horas aturdido $[\ldots]^{5}$

Según Cartwright, los síntomas de la "disestesia etiópica" habían sido correctamente identificados por los médicos de los estados abolicionistas, pero estos habían errado en el diagnóstico, creyendo que el embotamiento físico y mental que observaban en los esclavos era consecuencia de la violencia y la crueldad de la esclavitud. Nada más lejos de la verdad, según Cartwright, quien había observado que:

La disestesia etiópica es mucho más prevalente entre los negros libres que viven sin amo que entre los esclavos de nuestras plantaciones y ataca solo a los esclavos a los cuáles se permite vivir como viven los negros libres en cuanto a la dieta, las bebidas, el régimen de ejercicio $[\ldots]^{5}$

El estudio de Cartwright demuestra que la disestesia etiópica es una enfermedad grave, pero por suerte relativamente fácil de curar:

La mejor manera de estimular la piel es, primero, lavar al paciente a fondo con agua tibia y jabón; a continuación, untarlo completamente con aceite y hacer penetrar el aceite en la piel a latigazos administrados con una cinta ancha de cuero; a continuación hay que poner al paciente a trabajar al aire libre y al sol en un trabajo duro que le obligue a expandir los pulmones, como por ejemplo partir leña o serrarla $[\ldots]^{5}$

Hay que combinar este tratamiento con el reposo suficiente, la mejora en la bebida y la comida y asegurándose de que el paciente se lava adecuadamente antes de ir a dormir y duerme suficientemente abrigado. 
En el mismo artículo, Cartwright describe clínicamente la que según él era una segunda enfermedad mental que afectaba únicamente a la población negra. La denominó "drapetomania" (del griego drapetes que significa "fugitivo") y la definió como el deseo imperioso que manifiestan ciertos esclavos a huir de la plantación incluso corriendo el riesgo de perder la propia vida. La causa del transtorno es, siempre según Cartwright, la falta de equilibrio con que son disciplinados estos esclavos: sus amos o bien son demasiado crueles o bien demasiado indulgentes. El tratamiento es encontrar la correcta medida de amor y de miedo que mantenga al esclavo en sumisión sin violentarlo más de lo estrictamente necesario.

Cartwright no trabajaba en solitario ni aislado de la propia comunidad científica cuando describe estas dos nuevas enfermedades y las bautiza con términos científicos derivados del griego. Era el presidente de una comisión científica formada, además de por él mismo, por los doctores Copes, Williamson y Browning. La Asociación Médica de Luisiana, había encargado a estos cuatro médicos de prestigio que estudiaran las enfermedades y las peculiaridades anatomo fisiológicas de la población negra que era mantenida esclava en los estados del sur en contra de la voluntad de los estados abolicionistas norteños de los actuales EE. UU. Todos los estados norteños habían aprobado decretos de emancipación de esclavos entre los años 1780 y 1804 y el imperio británico había abolido la esclavitud legal en 1833 (Slavery Abolition Act). El estudio de Cartwright y otros intenta justificar en términos médicos, un sistema de producción y de vida basado en la explotación que parecía estar llegando a su fin. Un año antes de que la comisión presentara su informe (en 1850), los estados abolicionistas firmaban un compromiso con los estados esclavistas para evitar la división política de la Unión, pero finalmente las tensiones se hicieron insoportables y se produjo la guerra civil norteamericana. El año 1865 (catorce años después del informe Cartwright), los estados abolicionistas ganaron la guerra y el presidente Lincoln anunció el fin de la esclavitud legal en los EE. UU. (enmienda constitucional número 13).

Podemos pensar que el año 1851 y esta forma de racismo pseudocientífico de Cartwright nos quedan lejos, pero la medicalización de los problemas sociales ha tenido continuidad desde entonces hasta nuestros días en cuanto al intento de justificar la posición social de desventaja del conjunto de la población afroamericana en los EE. UU. En el año 1970, apenas dos años tras el asesinato de Martin Luther King, el neurocirujano Vernon $\mathrm{H}$. Mark y el psiquiatra Frank R. Ervin, ambos profesores de la facultad de medicina de la universidad de Harvard, publicaron conjuntamente un libro que fue un éxito de ventas: "La violencia y el cerebro". El libro explica en detalle las experiencias de estos dos médicos en el tratamiento de personas que tenían ataques de violencia y comportamiento antisocial. Se trataba de localizar con la máxima precisión por medio de unos electrodos metálicos la zona del cerebro que se activaba durante los ataques y de hacer a continuación una exéresis quirúrgica de las zonas afectadas. En la mayoría de los casos la zona activada durante los ataques correspondía al sistema límbico (cerebro emocional) y la exéresis consistía a menudo en la extirpación de un núcleo cerebral denominado "amígdala", tras lo cual el paciente quedaba sin capacidad de enfadarse ni sentir emociones con normalidad. ${ }^{6}$ En colaboración con el Dr. William Sweet, jefe de neurocirurgia del Massachussets General Hospital, los doctores Mark y Ervin avanzaron la hipótesis de que la violencia que la mayoría de afroamericanos consideraban una reacción a la marginación y la pobreza que sufrían, era en realidad una disfunción del cerebro. En una carta conjunta publicada en la revista oficial de la Asociación de Médicos Americanos (J AMA), los doctores Sweet, Mark y Ervin defendían así su posición:

si las condiciones de los barrios marginales por sí mismas fueran la causa de los disturbios, ¿cómo se explicaría que la mayor parte de quiénes viven en estos barrios sean capaces de resistir la tentación de la violencia descontrolada? ¿Hay algo peculiar en el habitante violento del barrio marginal que lo distinga de su vecino pacífico? [...] 
Sería de gran interés descubrir qué porcentaje de los intentos de asesinato y de los asesinatos consumados durante los disturbios recientes fueron violencia gratuita [...] Es imperativo llevar a cabo una investigación intensa y hacer estudios clínicos de los individuos responsables de la violencia. El objetivo de estos estudios sería identificar, diagnosticar y tratar a las personas que tienen dinteles bajos para la violencia a fin de evitar que en el futuro sean causa de más tragedias. ${ }^{7}$

Tal como ocurrió en su momento con el Dr. Cartwright y sus ideas sobre la drapetomania y la disestesia etiópica, los doctores Sweet, Mark y Ervin, tampoco trabajan aislados de la propia comunidad científica ni social. El año 1973, el Dr. Sweet recibe una subvención de medio millón de dólares del Instituto Nacional de Salud Mental de los EE. UU. ( NIMH), para investigar la mejor manera de diagnosticar y controlar médicamente a las personas que cometen violencia gratuita y a los delincuentes reincidentes. El mismo año, los doctores Mark y Ervin reciben 100000 dólares del Departamento de Justicia de los EE. UU. con el mismo objetivo.

En un estudio del 2008, la investigadora de la facultad de derecho de Harvard, Amanda C. Pustilnik se pregunta:

¿Tiene algún sentido hablar de "la neurobiología de la violencia" o de la "psicopatología del crimen"? ¿Es posible encontrar a nivel fisiológico la causa que hace que en circunstancias similares una persona actúe de forma criminal y otra no lo haga? ${ }^{8}$

\section{Su respuesta es clara:}

Durante los últimos doscientos años, el derecho penal y la neurociencia han mantenido una relación que está destinada al fracaso y que a menudo ha tenido consecuencias trágicas. En la investigación actual se pueden identificar los dos errores principales responsables de los fracasos previos. El primero es la "localización", a saber, la pretensión de agrupar los diversos fenómenos que denominamos "violencia criminal" bajo una única entidad clínica y de localizar las áreas del cerebro responsables de esta disfunción. El segundo error es la "alterización", a saber, la suposición que las personas responsables de los crímenes violentos son biológicamente distintas de las personas normales. ${ }^{8}$

La medicalización de los problemas sociales es un fenómeno que afecta de forma creciente a las sociedades contemporáneas, en contextos sociales aparentemente tan alejados cómo son el de Noruega (estudio sobre el paro), los EE. UU. (neurobiología de la violencia) o Israel. El Dr. Daniel Filc, que además de médico es profesor del departamento de Política y Gobierno de la Universidad "Ben Gurion" en Beersheva (Israel), ha analizado durante un período de cinco años las dos principales publicaciones científicas de la asociación de médicos de este país: el Israel Journal of Medical Sciences (publicada en inglés) y la Ha'refuah (que se publica en hebreo). Entre 1990 y 1995, únicamente cinco artículos del total de 1430 artículos publicados en Ha'refuah, tuvieron en cuenta los aspectos sociales de la enfermedad; el porcentaje correspondiente en el Israel J ournal of Medical Sciences fue inferior al $1 \%$. Tras analizar los artículos dedicados a enfermedades que tienen un claro componente social, como por ejemplo, las infecciones infantiles en los territorios de la Palestina ocupada, y haber constatado que los autores ignoraban sistemáticamente este factor, Filc concluye:

La desigual distribución de poder en la sociedad contemporánea se encuentra reflejada y reproducida en la ideología médica [...] El medio fundamental para conseguirlo es la medicalización la cual incluye la desocialización de la enfermedad y la explicación de fenómenos sociales con términos médicos. ${ }^{9}$ 
Por "desocialización de la enfermedad" entiende Filc el proceso por el cual la ciencia médica, en lugar de reconocer las causas sociales de ciertas enfermedades, las sustituye por causas de tipo individual: por ejemplo, en lugar de reconocer que el estrés laboral a menudo es una reacción normal y sana a unas condiciones laborales hostiles, la ciencia médica se centra hoy en día en el individuo y se propone intervenir sobre él con propuestas farmacológicas o incluso quirúrgicas. Urge un estudio a fondo de la extensión del fenómeno de la medicalización de los problemas sociales en los diferentes contextos nacionales y culturales, y urge una toma de posición crítica al respecto que desenmascare la manipulación ideológica disfrazada de ciencia, y retorne al profesional sanitario y particularmente al especialista en salud pública al lugar que le corresponde: al lado del paciente, con particular atención por los más vulnerables.

\section{REFERENCI AS BI BLI OGRÁFICAS}

1. Kristensen P, Bjerkedal T, I rgens LM. Birthweight and work participation in adulthood. Int J Epidemiol. 2004;33(4):849-56. doi: 10.1093/ije/dyh111.

2. Phillips DIW, Handelsman DJ, Eirksson JG, Forsén T, Osmond C, Barker DJP. Prenatal growth and subsequent marital status: longitudinal study. BMJ .

$2001 ; 322: 771$.

3. Cheung YB. Commentary: Fetal origins of social situations? Medicalization of social life? Int J Epidemiol. 2004;33(4):856-7. doi: 10.1093/ije/dyh160.

4. Illich I. Medical Nemesis. London: Calder and Boyars; 1974.

5. Cartwright SA. Diseases and peculiarities of the negro race. New Orleans Medical Surgical J. 1851; 7:691-715.

6. Mason BJ . New Threat to Blacks: Brain Surgery to Control Behavior-Controversial Operations Are Coming Back As Violence Curbs. Ebony. 1973; February: 63-72.

7. Mark VH, Ervin FR, Sweet W. Role of Brain Disease in Riots and Urban Violence. JAMA. 1967;201:895.

8. Pustilnik AC. Violence on the Brain: a critique of neuroscience in criminal law [abstract]. Harvard Law School Faculty Scholarship Series. Harvard: Nellco; 2008.

9. Filc $D$. The medical text: between biomedicine and hegemony. Soc Sci Med. 2004; 59: 1275-85.

Teresa Forcades i Vila. Monastir de St. Benet 08199-Montserrat. Cataluña, España. Correo electrónico: teresa@benedictinescat.com 